Róża Kosińska, Piotr Woltanowski

\title{
RÉFORMES DU SYSTÈME DE FINANCEMENT DE LA PROTECTION SANTÉ EN POLOGNE
}

Le système de financement de la protection santé en Pologne continue à changer depuis 1989. Le système qui fonctionnait dans la République Populaire de Pologne, qui consistait à financer le secteur directement par les moyens budgétaires n'était pas adéquat dans la nouvelle situation économique. On lui a reproché particulièrement le manque d'analyse de l'effectivité des coûts et de l'efficience de l'activité des unités de prévention sanitaire, le fait de favoriser des investissements excessifs et irrationnels en construction de bâtiments dans le secteur de la santé publique, le manque de coordination dans la politique d'investissements, le fait de causer l'acquisition irrationnelle et sans contrôle des instruments et matériaux médicaux, l'effectivité insuffisante de l'administration, le manque d'une gestion financière rationnelle dans les hôpitaux et autres institutions des soins de santé, ainsi que leur endettement toujours croissant, l'augmentation continue des dépenses à titre de financement des médicaments subventionnés et le fait de forcer la participation du patient à couvrir les frais des soins médicaux. ${ }^{1}$ Depuis le 1 janvier 1999, on a introduit en Pologne les caisses des malades, ce qui devait rendre ce secteur autonome et indépendant des décisions politiques arbitraires, dictées partiellement par des groupes de pression et partiellement par le besoin de faire équilibrer le budget et de garantir l'augmentation prévisible des moyens publics destinés à cet objectif. Le principe de base de la réforme était d'admettre un système mixte budget-assurances pour subventionner les soins de santé. Le budget de l'Etat devait financer entre autres les programmes de la politique de santé ainsi que les programmes de prophylaxie, la lutte contre l'alcoolisme, la prévention de la toxicomanie et du SIDA. Seize caisses des malades régionales et la Caisse des Malades pour les fonctionnaires en uniformes, ont assumé la fonction des payeurs, en associant les assurées assujettis à l'assurance obligatoire ou volontaire. Presque tous les citoyens étaient assujettis à

1 Vide: Relation du Réprésentant du Gouvernement pour l'Introduction du Système Public des Assurances, une année après l'entrée en vigueur de la loi sur les assurances maladies, envoyée par le Maréchal du Parlement de la République de Pologne Maciej Plazynski, n²413, site Internet du Parlement de RP du 1 juin 2008, www.sejm. gov.pl 
l'assurance obligatoire. Ceux qui n'étaient pas assurés en vertu de la loi, pouvaient choisir une assurance volontaire. Les frais des personnes qui n'étaient pas assurées et ne pouvaient pas couvrir les frais des traitements médicaux, étaient couverts par les fonds de l'assistance sociale. Formellement, on garantissait aux assurés l'accès gratuit à presque tous les genres de services de santé. Dans les contrats stipulés par les Caisses des Malades avec les fournisseurs des soins de santé, on définissait les droits, les obligations et les responsabilités des sujets fournissant ces soins. Les assurés avaient le droit de choisir la Caisse des Malades à laquelle ils voulaient appartenir.

Le système du financement présenté ci-dessus a été modifié par la loi du 23 janvier 2003 sur l'assurance générale auprès du Fonds national de la santé2. Les caisses des malades ont été remplacées par le Fonds national de la santé formé de la Centrale et de 16 succursales de voïévodie, selon la nouvelle division territoriale de l'Etat. La protection de la santé est actuellement financée par les moyens publics et privés. Les plus importants sont les revenus provenant des cotisations accumulées dans le Fonds national de la santé. Les moyens provenant du budget national et des budgets des unités des autonomies locales jouent un rôle moins important. En plus, le système de protection de la santé perçoit les moyens pécuniaires privés, payés par les patients, par leurs établissements de travail, par les assureurs privés et par les organisations caritatives. Le système de l'assurance maladie qui fonctionne actuellement en Pologne est basée sur les principes de l'universalité et de leur caractère obligatoire. Le devoir de payer l'assurance maladie et son caractère universel résultent de l'article 68 alinéa 2 de la Constitution de la République de Pologne, qui garantit que les autorités publiques assurent de la même manière l'accès aux prestations et soins médicaux financés par les moyens publics à tous les citoyens, sans tenir compte de leur situation matérielle.

Depuis l'introduction du système de financement de la protection de la santé par l'intermédiaire des caisses des malades et du Fonds national de la santé, on observe une diminution nette du financement par le budget de l'Etat. Ceci implique la limitation de la portée du financement des programmes de la politique de santé, des programmes d'action publique pour la protection et la restructuration, ainsi que le passage d'une part des compétences de la protection de santé et de santé publique administrée par le gouvernement au financement dans le cadre général de l'assurance maladie. En même temps, il y a une tendance a abaisser les dépenses patrimoniales destinées à la protection de la santé qui, en 2003, ne comprenaient que $60 \%$ des dépenses analogues de l'an 1999. ${ }^{3}$ Le non-investissement en locaux et équipement résulte du fait que les moyens obtenus grâce aux contrats ne sont

2 Journal Officiel $n^{\circ} 45$, position 391 avec mod.

3 S. Golinowska (red.) - Raport - Finansowanie ochrony zdrowia w Polsce - Zielona Księga, Warszawa 2004 
pas toujours suffisants pour couvrir les frais courants des procédures médicales. En Pologne, on ne destine à la protection de la santé qu'un peu plus de $6 \%$ du PIB, pendant que dans les pays de OECD on y destine en moyenne $8-9 \%$ du PIB. Le Fonds national de la santé, en disposant des moyens tellement limités, contracte les prestations en dessous de la demande, ce qui provoque des queues toujours plus longues, la limitation des prestations dues aux patients et la croissance du phénomène du "marché gris" des prestations médicales (prestations privées, entièrement payées par le patient, mais dispensées en utilisant les appareils et les équipements payés avec de l'argent public). Les moyens destinés aux rémunérations constituent un élément base de la structure des frais de la plupart des établissements des soins médicaux - l'augmentation de salaires, qui est indispensable, approfondira encore la crise financière dans ce secteur.

Du front à l'inefficacité du système public de financement de la protection de la santé, on observe le développement du marché des assurances maladie privées. Elles prennent forme des "paquets" d'abonnements, offerts par les entreprises fournissant des soins médicaux, ainsi que la forme des assurances maladie sensu stricto. Les premiers embrassent actuellement 400-500 milles salariés et comprennent les prestations de la médecine de travail ainsi que les prestations ambulatoires. La valeur du marché de ces abonnements est estimée à 200-300 millions de zlotys polonais4. En Pologne, ce n'est que le commencement des paquets des assurances maladie et leur valeur de marché est sensiblement plus basse par rapport aux abonnements médicaux. La barrière principale du développement des assurances maladie en Pologne est à l'heure actuelle le manque des régulations juridiques qui rendent possible cette activité, le niveau matériel peu élevé de la société, ainsi que le manque de conscience sociale limitant la disponibilité à prévenir le risque des dépenses futures pour la santé. D'autres facteurs qui influencent d'une manière négative le développement des assurances sont : le manque d'espace pour l'activité assicurative - le système universel garantissant (en théorie) toutes les prestations au niveau mondial le plus élevé, l'offre du "marché gris", le manque de réseau efficace des fournisseurs des soins de santé et le manque de régulations juridiques transparentes qui appuient le développement du marché.5

Les directions de la réforme actuellement initiée, qui concerne le financement de la protection de la santé, ont été déterminées par les projets antérieurs irréalisés. Dans Analyse de la situation et programme des changements de système dans le secteur de la protection de la santé en Pologne $e^{6}$ on postulait de définir le panier des

Informations plus détaillées: Rola dobrowolnych ubezpieczeń zdrowotnych w kontekście zmian systemowych sektora opieki zdrowotnej w Polsce, dokument zespołu roboczego ds. ubezpieczeń zdrowotnych Polskiej Izby Ubezpieczeń, passim.

5 Ibidem, p. 3

$6 \quad$ M. Boni, A. Kraszewski et M. Grajek, Analiza sytuacji i program systemowych zmian w sektorze opieki zdrowotnej w Polsce, le site Internet du Parlement de la République de Pologne du 1 juin 2008, www.sejm.gov.pl 
soins garantis, d'introduire de petits paiements pour les prestations dans le système public, et d'introduire les assurances volontaires supplémentaires. Ces propositions ont été développées et précisées dans le document Augmentation du rôle des assurances maladie volontaires dans le financement de protection de la santé en Pologne. ${ }^{7}$ Dans le projet des Programmes de Santé des Travailleurs ${ }^{8}$ on postulait de tels changements dans le système des impôts, pour que les assurances maladies de corporations puissent êtres considérées comme dépenses déductibles. Le projet du Syndicat Professionnel Polonais des Médecins Système Rationnel de protection de

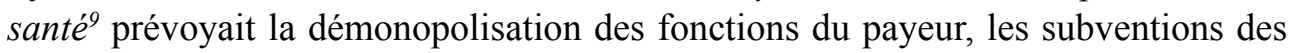
soins médicaux, l'élaboration d'un panier des prestations garanties et des assurances privées. Le projet de la loi sur le financement des prestations des soins de santé ${ }^{10}$ prévoyait l'existence des fonds de santé autonomes, possédant la personnalité juridique. Initialement, on ne pensait qu'aux institutions nationales, agissant sur des territoires définis, mais qui depuis l'an 2007 auraient la possibilité d'agir sur tout le territoire de la République de Pologne. Depuis l'an 2007 il existerait aussi la possibilité de créer des fonds de santé de caractère privé, agissant à la base de la loi et du code des sociétés commerciales, en tant que sociétés par actions. Le document Assurances non publiques en Polognell indiquait la nécessité d'introduire les assurances de santé non publiques, de définir le panier des prestations garanties et de standardiser les procédures médicales. Les postulats semblables ont été présentés par un groupe d'experts convoqué par le Médiateur. ${ }^{12}$ Le Sommet Blanc recommandait l'introduction du paiement d'une petite somme pour une partie des soins de santé et des prestations auxiliaires ${ }^{13}$, la création d'un panier positif des prestations garanties, l'introduction d'un système d'assurances privées supplémentaires complémentaires, et dans le futur, une concurrence libre des assureurs, ainsi que l'égalisation des obligations des agriculteurs et des autres assurés. ${ }^{14}$

Les propositions présentées, ainsi que le paquet des projets de loi qui procède actuellement au Parlement, et dont l'objectif est d'introduire une nouvelle réforme de protection de la santé ${ }^{15}$, devaient favoriser la réalisation de trois objectifs principaux:

Zwiększenie roli dobrowolnych ubezpieczeń zdrowotnych w finansowaniu opieki zdrowotnej w Polsce, le site Internet du Parlement de la République de Pologne du 1 juin 2008, www.sejm.gov.pl

8 A. Jacaszek, Pracownicze Programy Zdrowotne, document de la Confédération des Employeurs Polonais

9 Appuyé entre autres par la Chambre Nationale de Commerce polonaise (Krajowa Izba Gospodarcza) et par le Centre Adam Smith

10 Élaboré sous direction du ministre de la santé précédent - prof. Z. Religa

11 Niepubliczne ubezpieczenia zdrowotne w Polsce, document de Chambre d'Assurances Polonaise, le site Internet de la Chambre d'Assurances Polonaise du 1 juillet 2007, www.piu.org.pl

12 Site Internet du Médiateur (Rzecznik Spraw Obywatelskich) du 1 juillet 2007, www.rpo.gov.pl

$13 Y$ compris les frais du logement et de l'alimentation des patients

14 Reccomandations de la conférence du Sommet Blanc du 19 mars 2008, "Puls", revue mensuelle de la Chambre Régionale des Médecins à Varsovie, $2008 n^{\circ} 4$, page 6 et les suivantes.

15 Projet de la loi normes introductives concernant les lois en matière de protection de la santé - bulletin $n^{\circ} 294$, projet de la loi sur les établissement des soins médicaux - bulletin $n^{\circ} 284$, projet de la loi sur les assurences 
- Augmentation du niveau de financement des soins de santé,

- Définition de la portée de la protection de la santé financée par les moyens publics,

- Introduction des mécanismes motivant les fournisseurs des prestations à rationaliser les dépenses couvertes par les moyens pécuniaires publics (y compris un contrôle efficace et la définition de la portée des soins de santé qui sont dus dans le cadre de l'assurance maladie).

Entre les propositions discutées actuellement, ayant pour but d'augmenter le niveau de financement des soins de santé il faut énumérer:

- Introduction du système des prestations supplémentaires,

- Augmentation des cotisations de l'assurance maladie,

- Introduction du copaiement de la part des patients.

Parmi les projets de changement dans le système de financement de la protection de la santé, c'est l'introduction dans le système des assurances maladie de la possibilité de stipuler les contrats de l'assurance maladie en conformité avec la loi de l'activité assicurative, c'est-à-dire la stipulation d'une seconde assurance maladie volontaire, qui a la meilleure chance de réussite. Dans le projet de la loi, on a défini deux genres d'assurances maladie volontaires: assurance maladie supplémentaire et assurance maladie complémentaire.

Dans le cadre de l'assurance maladie supplémentaire, l'établissement d'assurances privées devrait fournir à l'assuré l'accès aux soins de santé garantis, pendant que l'assuré devrait renoncer à l'assurance parallèle dans le Fonds national de la santé. Il semble que les assurances supplémentaires, au cas où elles seraient introduites, ne pourraient pas jouer de rôle d'une source supplémentaire du financement des soins de santé qui leur était attribué par les auteurs de ce projet. Les personnes le plus exposées à la nécessité de profiter des soins de santé ne choisiraient point cette forme d'assurance, vu le montant des cotisations qu'ils auraient dû payer. En cas des personnes à petit risque assicuratif, les sommes provenant des cotisations assicuratives resteraient dans l'établissement de l'assureur sans être destinées aux fournisseurs des soins de santé. Au cas où l'on accepterait la possibilité de déduction de la cotisation de l'impôt ou de la cotisation obligatoire, c'est en pratique le budget national ou l'assureur public qui en aurait supporté les frais. A côté de ce phénomène de segmentation subjective du risque, on observerait probablement un autre phénomène: les assureurs se limiteraient à des procédures rentables en laissant les autres activités à l'assureur public. 
En cas de l'assurance maladie complémentaire, l'établissement des assurances devrait permettre à l'assuré l'accès aux soins de santé non garantis ou bien le financement d'une partie des frais des soins partiellement garantis, selon des principes définis par la loi. En cas d'un contrat de l'assurance maladie complémentaire, la prestation de l'établissement d'assurances consisterait à couvrir la différence entre le prix de la prestation financée par les moyens pécuniaires publics et le prix de la prestation établi par le fournisseur des soins de santé. Il faut toutefois souligner qu'il y a déjà en Pologne des assurances qui fonctionnent d'une manière semblable et la régularisation de ce segment du marché, toute utile qu'elle soit, n'augmentera probablement pas l'intérêt pour ce type d'assurances. La pratique actuelle de leur fonctionnement montre que l'assureur ne sera pas intéressé à la qualité élevée des prestations qu'il offre, particulièrement en ce qui concerne le remboursement des frais supportés par les établissements des soins de santé pour les traitements médicaux des patients, mais destinera plutôt les fonds à la promotion de ses services à travers la publicité.

On peut avoir l'espoir en l'augmentation des moyens provenant des cotisations et du budget de l'Etat. Selon les communications annoncées par le Président du Conseil des Ministres, la cotisation pour l'assurance maladie sera augmentée en 2010 de 9 a $10 \%$, et les dépenses budgétaires pour la protection de la santé devront augmenter de 5 milliards de zlotys polonais. ${ }^{16}$ Toutefois, les informations de ce type énoncées par les chefs des gouvernements précédents contribuent à faire refroidir l'optimise exagéré. Hélas, il ne faut pas penser à la réussite d'une décision socialement impopulaire, comme par exemple celle de copaiement limité de la part des patients. Et pourtant, l'acceptation de cette solution garantirait non seulement l'affluence des moyens au système, mais surtout influencerait une certaine rationalisation des comportements des patients et des fournisseurs des soins de santé. Il faut souligner que les montants de copaiements devraient être bas, et le système de l'aide financière (fonctionnant selon le principe de remboursement) devrait faire disparaitre les difficultés à obtenir les prestations accessibles aux personnes possédant moins de ressources.

Le montant des moyens destinés au financement du système de protection de la santé exige une définition de la portée des soins de santé auxquels on a le droit dans le cadre de l'assurance maladie. C'est le Tribunal Constitutionnel qui l'a souligné dans la sentence du 7 janvier 2004, en disant que le droit sur lequel est fondé le système de protection de la santé devrait préciser d'une manière univoque la portée des prestations financées par l'assuré. Selon les exigences constitutionnelles (art. 68 alinéa 2), la loi devrait définir d'une manière positive ou négative le panier des soins garantis. Dans le projet de la loi sur le changement de la loi sur la prestation 
des soins de santé financés par les moyens publics et des autres lois, on présume que le panier négatif sera introduit en forme d'une disposition du Ministre de la Santé, ce qui est motivé par la "possibilité de réagir d'une manière élastique aux changements dans le domaine du développement de la science et de la médecine". Dans la loi du 27 août 2004 sur la prestation des soins de santé financés des moyens publics (actuellement en phase de rédaction), on indique dans l'article 17 les prestations exclues du financement public. Toutefois, c'est une régulation fragmentaire qui ne définit pas la façon de prendre des décisions sur les prestations des soins de santé qui ne seraient pas couvertes dans le cadre de l'assurance maladie obligatoire, ce qui est une condition indispensable de transparence du financement des prestations des soins de santé. C'est l'Agence d'Evaluation des Technologies Médicales qui doit s'occuper de la qualification des prestations de soins de santé en tant que partiellement garantis ou non garantis. Selon le projet, elle le fera d'office ou sur demande. Cette Agence fonctionne actuellement à la base de la normative du Ministre de la Santé et elle doit effectuer la valutation des prestations des soins de santé, examiner les demandes concernant la qualification des prestations des services de santé, et son Conseil de Consultation doit recommander au Ministre de la Santé les décisions à prendre, en ce qui concerne la collocation d'une prestation donnée dans le panier des prestations de soins de santé. Le Ministre de la Santé est tenu par la recommandation émise au non-financement de la prestation donnée. Le projet définit aussi les principes de fonctionnement, l'organisation et les tâches de l'Agence d'Evaluation de la Technologie Médicale. Celle-ci sera une unité organisationnelle nationale possédant la personnalité juridique, surveillée par le ministre compétent pour les affaires de la santé, agissant à la base de la loi et du statut octroyé par la disposition du Ministre de la Santé. La norme de l'article 4 du projet de la loi a une importance essentielle, en obligeant le Ministre de la Santé à émettre une disposition contenant la première liste des prestations garanties et non garanties 6 mois avant l'entrée en vigueur de la loi.

Le panier des prestations consultées actuellement avec les milieux médicaux est lié à l'introduction d'un système des groupes uniformes des patients, qui rationalise les dépenses publiques dans cette matière. Le payeur effectuera les règlements des comptes avec l'hôpital selon les tarifs forfaitaires attribués aux cas des maladies qui restent uniformes du point de vue des frais, de la diagnose ou des procédures médicales effectuées, et forment des groupes précis dénommés GUP - groupes uniformes des patients. Le calcul des frais de traitement du patient consiste à le classer selon les procédures effectuées et à le mettre dans un groupe défini. Il est basé sur les procédures du rang le plus important, sur les procédures supplémentaires, la diagnose principale et coexistante, l'âge du patient et la durée de l'hospitalisation. Les avantages fondamentaux de ce nouveau système sont la simplification des principes du financement et leur corrélation avec les coûts réels de la prestation. En 
plus, l'introduction de ce système rend possible d'effectuer une analyse des frais crédible ${ }^{17}$.

Les changements dans l'organisation du système des assurances planifiés par le Ministère de la Santé, comprennent l'introduction d'autres payeurs des soins de santé, à part le Fonds national de santé (FNS), qui seraient financés avec des cotisations publiques, ainsi que la division du FNS en plusieurs assureurs concurrentiels. Comme dans le "Projet de Religa", on prévoit, à l'étape finale de la réforme (prévue pour l'an 2012), d'introduire sur le marché des assureurs privés. Il est pourtant difficile de partager l'opinion de Madame la Ministre de la Santé, qui souligne que le nouveau système permettra de s'éloigner des prestations de santé limitées. Laisser cette question aux assureurs comporterait une probabilité trop élevée du phénomène de segmentation subjective du risque qui a déjà été mentionné.

Pour garantir une surveillance efficace des assurances volontaires, la Commission de Surveillance Financière a été élargie, et comprend en plus le Ministre de la Santé ou son représentant. Vu l'introduction de nouvelles assurances maladie (à part les assurances obligatoires), le projet présume aussi la convocation d'une nouvelle unité - Bureau de Surveillance des Assurances Maladie, compétente pour le contrôle du système de l'assurance maladie générale et l'activité des établissement des assurances concernant les assurances maladie volontaires. Sa tâche consistera à protéger les intérêts des patients et des assurés. La Surveillance de ce Bureau (conduite selon le critère de légalité, sincérité et intentionnalité) concernera l'activité du Fonds, des fournisseurs des prestations, des pharmacies (le remboursement des médicaments) et des établissements d'assurances qui mènent leur activité sur le territoire de la République de Pologne en fournissant des assurances maladie volontaires. Il semble que le projet de la loi sur les assurances maladie volontaires, qui est actuellement élaborée par le Parlement, exige encore certaines modifications. La Surveillance de la part du Président du BSAM ne sera pas, malgré l'attribution des compétences très étendues, très efficace en cas des pharmacies. Il est indispensable que cet organe possède la possibilité d'infliger des sanctions sensibles : en conformité avec l'article 188 du projet, au cas où la pharmacie n'éliminerait pas dans le délai prévu des irrégularités constatées, le Président du Bureau de Surveillance peut infliger une peine pécuniaire dont le montant équivaut à la rétribution moyenne de trois mois. ${ }^{18}$

La transformation préannoncée de tous les hôpitaux, sauf les cliniques et les unités du ministère, en sociétés de droit commercial a pour objectif de rationaliser les dépenses publiques grâce à la prévention de leur endettement dans le futur.

17 Cfr. J. Paszkiewicz, Jednorodne Grupy Pacjentòw - przewodnik po systemie "Menedżer Zdrowia" 2008, n 6, page 2 et suivantes

18 Dans une situation analogue, le Président du Bureau inflige au fournisseur des soins de santé une peine pécuniaire dont le montant équivaut à la valeur du contrat mensuel stipulé entre ces fournisseurs et le Fonds, qui regarde l'irrégularité en question. 
Les irrégularités dans la gestion économique et financière de ces centres doivent être découvertes suite à l'audit effectué avant leur passage sous la gestion des autonomies locales. Il y a ici une question controversée, celle du désendettement des établissements autonomes publics des soins de santé. Ce processus doit se dérouler d'une manière progressive et graduelle et il devrait consister à convertir leur endettement en papiers de valeur à long terme. ${ }^{19} \mathrm{C}$ 'est pourquoi on doit former un fonds de restructuration. Les autonomies locales, après avoir repris les hôpitaux, devront décider de leur privatisation..$^{20}$ En plus, le projet de la loi Normes introductives de la loi sur la protection de santé prévoit l'acceptation d'une solution qui introduise le mécanisme assurant l'intérêt constant de l'unité de l'autonomie locale pour la situation économique de l'établissement autonome public des soins de santé. Dans la loi sur les finances publiques on doit introduire une clause stipulant que dans le cas de l'unité de l'autonomie locale qui fonde un établissement autonome public des soins de santé, le montant total des dettes d'une telle unité ne peut pas dépasser à la fin de l'année budgétaire $60 \%$ du revenu total produit par cette unité au cours de la même année budgétaire, diminué du montant des charges de cet établissement exigibles à la fin de l'année budgétaire.

Pour éliminer les abus qui regardent la prescription des médicaments remboursés, le ministère de la santé prévoit l'informatisation entière du système de remboursement. Les changements concernant cette matière deviennent une charge pour les médecins, qui auraient de nouvelles obligations à remplir, mais, ce qui est plus important, ils seraient très coûteux pour les établissements des soins de santé, qui devraient être munis de lecteurs spéciaux des cartes magnétiques et de programmes appropriés. Cela a provoqué des contestations de la part des médecins. De même, les solutions temporaires consistant à introduire de nouveaux modèles d'ordonnances avec le système de sécurité divisé en six niveaux, imprimés par la société Entreprise Polonaise des Papiers de Valeur (Polska Wytwòrnia Papieròw Wartosciowych), ne trouve pas l'acceptation. ${ }^{21} \mathrm{C}^{\prime}$ est tout à fait compréhensible, vu que selon le projet du ministère, les frais des changements en question passeraient à la charge des médecins qui auraient dû acheter des formulaires d'ordonnances, tandis que l'introduction des systèmes de sécurité n'éliminerait pas les abus qui consistent avant tout à émettre des ordonnances d'une manière fictive par certains médecins, au nom des personnes ayant droit au remboursement total, ce qui porte à extorquer la somme remboursée. En plus, il est assez facile d'éliminer ce problème à travers le contrôle de la personne qui achète les médicaments remboursés prescrits par un médecin donné, de celui

\footnotetext{
19 Selon les propositions du Ministère de la Santé - les obligations du Trésor. L'Association des Managers des Soins de Santé STO-MOZ dans la déclaration du 14 mars 2008 propose l'émission des obligations de la part des unités de l'autorité locale.

20 II y a une analyse intéressante de ses propositions dans: E. Szarkowska, M. Stańczyk, Samorządy zdecydują o przyszłości zadłużonych szpitali, „Puls Medycyny” 2008, n 9, p. 4-5

21 Cfr. M. Stankiewicz, Recepty niczym banknoty, "Gazeta Lekarska” - Pismo Izb Lekarskich 2008, nº p. 18
} 
pour lequel le médicament est prescrit et de celui qui les vend. Il existe une question encore plus urgente, celle d'élaborer des méthodes transparentes qui permettent de vérifier la liste des médicaments remboursés. Actuellement, elle rend possible l'activité de lobbing de la part des entreprises pharmaceutiques. La portée de cette activité est énorme. La procédure en cette matière devrait être strictement formalisée et surveillée: il est inadmissible que sur ces listes existent des médicaments qui sont hors production ou bien des produits qui ont été retirés du marché (cette année on a supprimé de la liste 140 produits de ce genre), que les demandes d'inscription sur la liste attendent des années ${ }^{22}$, ainsi que l'inscription des médicaments qui ne possèdent ni recommandation ni valutation convenables.

Pour conclure, il faut souligner que les réformes actuelles introduites dans le système du financement du secteur de la santé doivent constituer un élément d'un plan plus large, échelonné sur plusieurs années. Il est nécessaire d'établir d'une manière claire un modèle-cible du financement ainsi que l'indication des délais de l'introduction des changements successifs, ce qui rendra possible une discussion constructive sur les principes préalables de la réforme, éliminera le manque de sûreté et permettra de préparer au changement les travailleurs du secteur de protection de la santé. Les projets des lois qui déterminent les cadres juridiques du futur système devraient être formulés dans le Ministère de la Santé, et non présentés par les députés. Cela limitera l'influence des entreprises des assurances et des propriétaires des pharmacies dont les résultats sont déjà plus que visibles à la lecture des projets. 


\section{Streszczenie}

Przeprowadzane od 1999 r. głębokie reformy instytucjonalne systemu finansowania ochrony zdrowia nie rozwiązały problemu niedofinansowania - w Polsce na opiekę zdrowotną przeznaczanych jest nieco ponad 6\% PKB. Skutkuje to narastającymi kolejkami, bezprawnym limitowaniem świadczeń medycznych dla pacjentów i rozwojem tzw. szarej strefy usług medycznych (świadczeń prywatnych, pełnopłatnych, jednak z wykorzystaniem aparatury medycznej zakupionej z pieniędzy publicznych). Procedowany w Sejmie pakiet projektów ustaw służyć ma zwiększeniu poziomu finansowania świadczeń opieki zdrowotnej, określeniu zakresu finansowanej z pieniędzy publicznych ochrony zdrowia (projekt tzw. koszyka świadczeń gwarantowanych jest konsultowany obecnie ze środowiskami medycznymi) i wprowadzeniu mechanizmów motywujących świadczeniodawców do racjonalizacji wydatków (w tym skutecznej kontroli i określenia zakresu świadczeń opieki zdrowotnej przysługujących w ramach ubezpieczenia zdrowotnego). Przekształcenie wszystkich szpitali poza klinicznymi i resortowymi w spółki prawa handlowego również ma na celu racjonalizację wydatków publicznych poprzez zapobieżenie ich zadłużaniu się w przyszłości. Aby wyeliminować nadużycia w zakresie wypisywania leków refundowanych resort zdrowia planuje docelowo pełną informatyzację systemu refundacji. 